\title{
La elaboración de interpretaciones en evaluación clínica
}

\author{
The development of interpretations in clinical evaluation
}

\author{
Carmelo Ibáñez Aguirre ${ }^{1}$ \\ Universidad del País Vasco, España
}

(Rec: julio 2012 - Acep: abril 2013)

\begin{abstract}
Resumen
Este trabajo de revisión teórica tiene como objetivo estudiar las cogniciones interpretativas del evaluador clínico. Se investiga analíticamente la naturaleza y requisitos científicos de las cogniciones y metacogniciones que componen la tarea de interpretar. Se analizan las inferencias de distinto nivel implicadas, sus posibilidades y alcance, así como sus limitaciones, errores y sesgos principales. Finalmente, para orientar la mejora de la calidad de las interpretaciones que ha de efectuar el clínico, se aportan algunas recomendaciones de carácter práctico. Palabras clave: interpretación psicológica, evaluación clínica, cogniciones del evaluador, sesgos
\end{abstract} interpretativos.

\begin{abstract}
The objective of this theoretical review paper is to study the cognitive interpretations of the clinical evaluator. This study investigates the analytical characteristics and scientific requirements of cognition and metacognition which comprise the task of interpreting. We analyze the inferences of different levels involved, their possibilities and extent, as well as their limitations, errors and biases. Finally, we provide some practical recommendations in an effort to guide and improve the quality of the interpretations made by the clinician.

Key words: Psychological interpretation, clinical evaluation, the evaluator's cognitions, interpretive biases.
\end{abstract}

\footnotetext{
1 Correspondencia a: Carmelo Ibáñez Aguirre, Avda. de Tolosa, 70. San Sebastián-20018. Email: Carmelo.ibanez@ehu.es. Teléfono: 34943015638. Fax: 34-943015670. Facultad de Psicología, Universidad del País Vasco, España.
} 


\section{Introducción}

La actividad del evaluador en la clínica se caracteriza por su alto grado de complejidad. Desde las percepciones iniciales que otorgan significado a los estímulos, la interpretación impregna todo su quehacer con creciente grado de compromiso conforme avanza el proceso evaluador. Las reflexiones cognitivas que siguen son de carácter epistemológico (Romo, 2008), se plantean al margen de las interpretaciones estandarizadas que acompañan a las pruebas cuantitativas (nomotéticas); no indagan en algoritmos o pasos interpretativos predeterminados, sino en heurísticos. El interés principal se centra en el proceso subyacente a la interpretación y la necesidad de revisar, por tanto, la praxis cualitativa (idiográfica), intuitiva y creativa, que está en el núcleo de la actividad del evaluador clínico. Este trabajo quiere conectar con el interés de la psicología cognitiva por aclarar los problemas del conocimiento y transitar el camino que abren las metacogniciones para el acceso a las interpretaciones y su génesis (Efklides, 2008; Ibáñez, 2003; 2005; Ibáñez y Maganto, 2009; Pintrich, 2002).

Este último objetivo es tanto más interesante en cuanto que, a diferencia de la evaluación empírica de la eficacia de los tratamientos psicológicos basada en la evidencia que ofrece el criterio de su efectividad, en evaluación rechazamos como prioritario este enfoque. Para contrastar la eficacia de las evaluaciones, en su lugar seguimos el modelo de aproximación "a priori" a la evaluación (Ibáñez, 1997). Motivo por el cual la responsabilidad y el grado de implicación del control que ejerza el evaluador sobre su propia praxis han de considerarse, hoy por hoy, decisivos. Así, por ejemplo, está por confirmar la eficacia y efectividad, así como el respaldo de la evidencia con que cuentan las principales guías, como las GAP a las que se aludirá más adelante.

Si una de las formas de definir la inteligencia dice que es "la capacidad de suscitar, dirigir y controlar las operaciones mentales" (Marina, 1996, p. 250), la meta del evaluador inteligente será suscitar, dirigir y controlar inteligentemente las interpretaciones que efectúe. Interpretar constituye una competencia sustantiva para el evaluador clínico. Inferir es el proceso mediante el cual se recoge y combina información a efectos de formular un juicio (Kaslow, 2004). Es necesario conocer los mecanismos, posibilidades y limitaciones que conlleva la interpretación para preservar la condición científica, la objetividad de las elaboraciones y conclusiones y poder controlar los errores y sesgos que sobre todo en este ámbito de cogniciones amenazan el buen hacer del clínico.

Se trata de una cuestión sólo en apariencia teórica, pues además de la componente asociada a la "episteme" posee una vertiente práctica en el ámbito aristotélico de la "phronesis" (sabiduría práctica, prudencia). Esta última atañe a las pautas sobre cómo actuar para mejorar en situaciones específicas, clínicas en nuestro caso. Más en concreto, refiere a la manera como se acierta a combinar entendimiento (saber especializado), experiencia y capacidad de leer de forma correcta las situaciones profesionales que se le plantean al clínico.

El modelo constructivista del conocimiento científico (Rodríguez Rebustillo y Bermúdez, 2004) enfatiza en la etapa de interpretación que sigue a las de exploración y reestructuración del problema estudiado. Más específicamente en lo que concierne a la formación del clínico cognitivo-conductual, BennettLevy (2006) ofrece un modelo organizado en los tres sistemas: declarativo, procedimental y reflexivo. Es de especial interés el segundo, pues atañe al "cómo" y "cuándo" de la aplicación de esquemas, así como a la actitud del clínico.

A continuación, empezaremos por estudiar la naturaleza de las interpretaciones, distinguiéndolas de las inferencias del razonamiento lógico. Tras apuntar su utilidad en la evaluación clínica, se anotarán los distintos niveles que adopta y los riesgos cognitivos que conlleva interpretar, con el fin de minimizarlos. Una vez diferenciadas las interpretaciones puntuales de las globales, con sus respectivas funciones específicas, se marcarán algunas pautas o recomendaciones que favorezcan la calidad de las interpretaciones. Estas pautas pueden ayudar a mejorar la formación no sólo de las habilidades cognitivas necesarias para evaluar en clínica, sino también las metacognitivas, que incluyen el control de los propios procesos cognitivos y la autorregulación (Nisbet y Shucksmith, 1986; Pintrich, 2002).

\section{El proceso de evaluación}

Aunque en la evaluación clínica se empleen con preferencia teorías y metodologías empíricas de inferencia mínima, desde la complejidad de la tarea, la necesidad de interpretar es obligada. Una de las razones que lo abonan es que, para su debido análisis las conductas problemáticas han de ser investidas de teoría; es la relación de constructos con los problemas. Estos tienen a menudo causas múltiples, además, varían en 
combinación con otras conductas, son idiosincrásicas y están determinadas por interacciones con el entorno sociocultural, así como con variables intra-individuales cognitivas, afectivas, motivacionales ( $\mathrm{O}^{\prime}$ Brien y Haynes, 1997).

Las tareas principales a realizar a lo largo del proceso de evaluación se resumen en tres: (a) acopiar información y datos empíricos, esto es, acerca de hechos evidentes; (b) teorizar sobre ellos, pasando de la formulación de hipótesis a contrastarlas y elaborar los datos válidos; (c) extraer conclusiones prácticas, lo que en particular conlleva trabajar cogniciones referidas a síntesis, interpretaciones e integraciones. Aunque la interpretación esté presente en todos los momentos del proceso evaluador, la intensidad interpretativa es mayor en la fase de sistematización final asociada a la emisión de juicios diagnósticos y toma de decisiones (Fernández-Ballesteros et al., 2001; Godoy y Gavino, 1999; Patel y Groen, 1992).

La naturaleza de las interpretaciones varía, pasando de ser meramente descriptivas, por ejemplo, las referidas a las puntuaciones obtenidas en los test, escalas, etc., a más complejas si atañen a la integración de datos, cuantitativos y cualitativos, provenientes de diferentes métodos y técnicas, tanto estadísticos como clínicos. En la fase hipotético-deductiva las inferencias razonadoras son las que corrigen cualquier arbitrariedad o especulación explicativa, lo que lleva a la verificación de las hipótesis transitando desde la prueba a la interpretación conclusiva. Se justifican así las interpretaciones iniciales (Dunkley, Blankstein y Segal, 2010). En último término, toda hipótesis es revisable. Nunca se puede afirmar tajantemente que una interpretación es justa y siempre se puede decir, o decir con más facilidad, cuándo es equivocada (Eco, 2012).

La interpretación tiene implicaciones múltiples durante el entero proceso de evaluación, unas son teóricas y otras aplicadas a los datos de observación y la subsiguiente elaboración de los mismos (Sandi-Urena, 2009). Cuanto más amplia sea la red de significados que el clínico vaya extrayendo, mayor será su capacidad de establecer nuevas relaciones e interpretaciones. La visión holística le ayudará a otorgar sentido a las mismas. Toda interpretación comienza con la necesidad de responder a preguntas que surgen ante la imposibilidad de comprender los fenómenos en sus propios términos (Evans, 1993; Levy, 1971). Y se plasma en un proceso cognitivo en el que se atribuyen características de una clase general a una individual que se considera incluida en la primera (Allport, 1977).
Las interpretaciones, que incluyen valoraciones, integraciones y juicios complejos, conllevan la transformación de lo molecular y aislado, en molar, holístico y contextualizado. Dichas transformaciones de tipo inferencial facilitan el acceso al significado de los comportamientos. Asimismo permiten su personalización, potenciándose de este modo el acceso a resultados de mayor relevancia y utilidad clínica (Prins et al., 2008).

Las cogniciones interpretativas atraviesan la totalidad del proceso de evaluación psicológica y atañen sobre todo al quehacer del evaluador: la recogida de información, el análisis, las síntesis de integración que preceden a las conclusiones en las que se apoyan los juicios clínicos y la toma de decisiones para la intervención... (Adams-Webber, 2003; McClelland, 2010). Son muchas y laboriosas las interpretaciones a realizar de parte del clínico.

Los síntomas psicopatológicos y las conductas problema en general requieren un análisis topográfico, en su caso la incorporación de interpretaciones de los resultados de mediciones (test, cuestionarios, escalas), la valoración del orden jerárquico y peso de cada uno de los resultados, la interpretación de la dinámica interactiva única entre ellos, su integración que va precedida de la visión holística y unitaria, la anticipación de las interpretaciones valorativas acerca de las consecuencias previsibles asociadas a las decisiones que se tomen, etcétera (Caro, 2011; Lee y Truex, 2000).

En las Guías del Proceso de Evaluación (GAP) (Fernández-Ballesteros et al., 2001) que pretenden registrar las principales tareas cognitivas a realizar por el evaluador psicológico a lo largo del correspondiente proceso, no se menciona la interpretación, con la única salvedad de la guía 36 ("El evaluador debe analizar e interpretar los datos de las pruebas y demás procedimientos de evaluación de acuerdo con las últimas normas, estándares y conocimientos disponibles"). Quizá se deba a que se dé por sobreentendido el componente de interpretación. De hecho, en mayor o menor medida se encuentra en cogniciones que ahí se mencionan como: identificar, comprender, analizar, explorar, averiguar, valorar, comprobar, comparar, distinguir, revisar, jerarquizar, sintetizar, integrar...; por supuesto, evaluar (Fernández-Ballesteros, Márquez, Vizcarro y Zamarrón, 2011).

En este trabajo se traen al primer plano las cogniciones de tipo inferencial, interpretativas, del proceso evaluador que con mayor énfasis se concentran en las fases de elaboración de datos, formulación de hipótesis y en las fases de integración que derivan en formulación del juicio diagnóstico y establecimiento de conclusiones 
con la correspondiente toma de decisiones. De forma que las consideramos una de las competencias generales de mayor importancia, asociada a las capacidades de inteligencia emocional y autonomía personal entre otras (Roe, 2002). En cualquier caso es necesario incorporar el objetivo de la mejora de dicha competencia a los programas de formación y cualificación de los psicólogos clínicos (Elman, Illfelder-Kaye y Robiner, 2005; Rodolfa et al., 2005).

\section{La interpretación}

Según Francis Bacon (1561-1626), "el hombre es un intérprete de la naturaleza". La conducta humana es acción significativa en el mundo, es decir, subjetiva y mental, con sentido (Yela, 1991). No cabe pensar en el proceso de construcción del conocimiento sin atender al fenómeno de la interpretación a muy distintos niveles. La interpretación es un proceso creativo que lleva a ampliar el conocimiento estableciendo conexiones nuevas y creando significados. La interpretación psicológica comienza cuando, en nuestro caso, el evaluador se pregunta sobre el significado de los datos, decide ir más allá de la estricta información que contienen y transformarlos cargándolos de inferencias. Lo cual supone redefinir y reestructurar, aportando descripciones alternativas conectadas con algún marco teórico de referencia del evaluador (Banyard, Cassells, Green, Hartland, Hayes y Reddy, 1995; James, Southem y Blackburn, 2004; Levy, 1971).

No se concibe conocimiento auténtico ajeno a la interpretación, en el nivel que corresponda. Comprender es captar significados. Bien es cierto que un concepto está abierto a varios significados. La evaluación conlleva discernir, tejer una red de significados y sentidos de carácter explicativo, personal y valorativo. "El concepto fundamental de la psicología humana es el significado y los procesos y transacciones que se dan en la construcción de los significados" (Bruner, 1990, 47). En el propio razonamiento ya existe, junto al deductivo, un componente de inferencia que establece la correspondencia entre los enunciados del lenguaje y el conjunto de reglas sintácticas (González Labra, 2011).

Los términos "significado" y "sentido" se utilizan como sinónimos, si bien el primero atañe más al "qué", el contenido; mientras que el segundo refiere al "para qué" o finalidad del comportamiento analizado. Para interpretar bien el sentido, además del contexto, se precisa conocer la intención, los valores y motivación, así como la finalidad u objetivo del comportamiento en cuestión.

En cuanto al evaluador científico, además de la sujeción al método y el respaldo de la teoría, es importante la consideración de aspectos humanos suyos tales como la actitud, los conocimientos previos, el talento, la creatividad y la capacidad de comprensión (Gabucio, 2004), sin los cuales resulta difícil imaginar que puedan producirse interpretaciones veraces.

Conocer equivale a traducir y reconstruir la realidad apoyándose en la observación y el razonamiento, pero completándolo con el salto adelante que propician los aportes de la intuición con sus hipótesis explicativas o de otro tipo que, una vez justificadas, cabrá incorporar al bagaje del conocimiento (Morin, 2001). La interpretación subsume distintas tareas cognitivas complejas de generación e integración, de absorción y transformación de la información (McClelland, 2010). Son procesos cognitivos todavía no bien conocidos que utilizan mecanismos específicos de representación mental que se activan especialmente en determinados momentos (AdamsWebber, 2003; Adams y Aizawa, 2010; Arias, 2012; Lee y Truex, 2000).

En psicología las informaciones y datos encierran un componente latente de ambivalencia en cuanto a su significado que es preciso resolver en función de las demandas del contexto en que se producen (Domingo, 2005). Así, por ejemplo, los sobreentendidos en la comunicación verbal son resultado de inferencias hechas por los interlocutores en función tanto del conocimiento compartido, como del clima de entendimiento y complicidad que existe entre ellos (Ibáñez, 2010).

En el lenguaje escrito el peso relativo del contexto a la hora de interpretar es menor que en el lenguaje oral, por lo que la información ha de ser más explícita a fin de ser bien entendida. Según sean los supuestos, el evaluador activará esquemas cognitivos diferentes para interpretar los mensajes e informaciones que le lleguen. En el primer caso será mayor la carga inferencial movilizada y la apelación a la intuición, debido a que, a diferencia de la comunicación verbal directa, no cuenta con el apoyo de códigos no verbales y la posibilidad de retroalimentación entre los agentes de la comunicación (Cormier y Cormier, 1994; Ibáñez, 2010).

Las inferencias que efectúa el evaluador le son útiles para distintos fines: determinar el alcance de una información, ampliar su contenido, codificarla y recodificarla, transformarla, atribuirle significado, inducir, deducir, abstraer, etc., útiles también en la abducción, esa forma de silogismo (conjetural y 
que empieza a ser creativa) cuya premisa mayor es evidente y la menor menos evidente o solo probable. Por supuesto la interpretación es asimismo un decisivo recurso para generar hipótesis, profundizar en el conocimiento construido, comunicarse con el paciente y acertar a personalizar los datos. Ahora bien, si las correspondientes interpretaciones no se concentraran en explicar lo observado, sino en tratar de explicar lo imaginado, se resentirá la validez de las mismas con riesgo de caer en la especulación (Butler, Champan, Forman y Beck, 2006; Hofer y Pintrich, 2002; Levy, 1971; Shinamura, 2000).

Es la teoría desde la que trabaja el evaluador clínico la que le suministra el código de leyes, signos y reglas útiles para comprender los comportamientos, las variables que éstos implican, su nomenclatura, qué interpretar y cómo, en suma, los recursos cognitivos y ciertas pautas para transformar la información en determinada dirección interpretativa. Esa actividad cognoscitiva de codificación y recodificación, de formación de conceptos, de despliegue de abstracciones, inducciones y deducciones, que conducen a reformulaciones transformadoras de la información de partida, es lo que se conoce como tarea interpretativa (Godoy, 1996; Levy, 1971).

La tarea de interpretación es necesaria, pero está erizada de riesgos para el clínico preocupado de realizar un trabajo que se caracterice por la objetividad y el rigor científico. Interpretar consiste idealmente en explicar y detectar el sentido o significado de un dato, profundizando en su comprensión y alcance como conocimiento completo. Sin embargo, "la interpretación suplanta a la explicación allí donde la explicación no tiene cabida" (Castilla del Pino, 1992, p. 1362). Aunque potencialmente múltiples para su incorporación a la ciencia, las interpretaciones asumen unos límites (Eco, 2009). Así, la interpretación (hermenéutica) facilita el acceso a la comprensión en profundidad, siempre y cuando las inferencias realizadas sean justificadas y no conlleven saltos intelectuales en el vacío, esto es, gratuitas especulaciones.

Para atenerse al marco científico las interpretaciones deben referirse a observaciones, ser ajustadas y precisas, fiables y válidas. En concreto la validez de constructo se refiere a la validez de las inferencias efectuadas sobre fenómenos no observables (constructos teóricos) en base a fenómenos observables o indicadores que se relacionan con aquéllos en el marco de alguna teoría o modelo teórico (Fernández-Ballesteros y Calero, 2004). Es indudable que se necesitan conceptos para pensar los datos.
Desde determinados marcos teóricos, como el de atributo o de rasgo, el evaluador toma los comportamientos del sujeto evaluado como signos de unos significados que el modelo de personalidad le presta y le ayuda a elaborar de modo congruente por cuanto que le permite establecer relaciones probabilísticas entre las variables estudiadas.

En términos generales cabe, pues, precisar que las interpretaciones nacen de conectar: (a) un determinado dato con otro u otros del mismo sujeto; (b) algún dato actual con otro u otros del pasado; (c) un dato que se asocia bien a un contexto u a otro; todos ellos contemplados desde un enfoque teórico u otro. De este modo, para obtener el significado de los datos es siempre necesario apoyarse, explícita o implícitamente, en conceptos pertenecientes a alguna teoría o modelo teórico (Ibáñez, 2003; Ibáñez y Maganto, 2009).

La interpretación parte de relacionar los datos de interés prioritario con otros asociados a éstos, así como con los del contexto y los objetivos que se hayan marcado. Las interpretaciones que vayan efectuándose afectan al conjunto de datos, con los que interactúan de manera múltiple y dinámica, originando cadenas de cambios e influencias innovadoras varias. Entre ellas la de catalizar, impulsar, acelerar o reconducir procesos que se sustancien en avance del conocimiento y depuración del mismo (León y Pérez, 2003).

Las interpretaciones que se ajustan a criterios de rigor científico, se seleccionan entre otras posibles. De modo que quien interpreta elige la opción que aparece más ajustada, decisión que no se lleva a cabo por capricho, ni de manera mecánica, sino mediando siempre un razonamiento que lo justifique. Las interpretaciones, por cuidadas que sean, no pasan de ser propuestas verosímiles, sólo quienes sufren intolerancia a la incertidumbre las convierten en certezas. Pero "toda interpretación dada como cierta es falsa" (Castilla del Pino, 1992, p. 1361).

Las inferencias, necesarias y presentes en todos los momentos del proceso de evaluación, se dan con más intensidad en el marco de unos determinados modelos teóricos que en otros; si se recurre más a métodos indirectos que directos; y si las variables analizadas son de nivel cognitivo en lugar de conductual o psicofisiológico. Es preciso, pues, mantenerse alertados contra el riesgo de inferir sin la necesaria contención y rigor (Godoy, 1996; Prins et al., 2008).

En todo momento el evaluador clínico ha de saber diferenciar lo que son evidencias empíricas de los supuestos hipotéticos con sus interpretaciones tentativas, 
ya que en ocasiones pueden ir demasiado lejos, indebidamente influenciadas por sus propios rasgos de personalidad (Fumero, Santamaría y Johnson-Laird, 2010). Han de diferenciarse las opiniones personales de aquellas interpretaciones bien fundadas, de contenido y nivel razonables, con su correlato de juicios diagnósticos debidamente sustentados. Así, por ejemplo, aquellas interpretaciones que haga el evaluador referidas a la generalizabilidad bien sea de las puntuaciones, elementos del test, temporalidad, situaciones, métodos en curso y dimensiones o variables evaluadas, conectarán estrechamente con las distintas clases de validez disponibles (Fernández-Ballesteros y Calero, 2004).

En general, en los distintos supuestos interpretativos el evaluador tratará de confirmar empíricamente la bondad de las posiciones adoptadas con el mayor rigor a su alcance. El descuido y la improvisación interpretativa precipitada han de sustituirse por un mayor grado de autoconciencia. El evaluador prestará atención a sus propias metacogniciones a fin de detectar las percepciones, pensamientos y sentimientos, que afecten negativamente a sus interpretaciones (Ibáñez y Maganto, 2009).

\section{Intuiciones y heurísticos}

Además de contar con las inferencias lógicodeductivas, inductivas, etc., se han de considerar, asimismo, las de tipo intuitivo-empático que por su naturaleza subjetiva exigen mayor autorregulación a fin de que puedan generar conocimiento válido (Tversky y Kahneman, 1984). Etimológicamente, intuición remite a "mirar con atención", lo que induce a una visión intelectiva por inspiración, con percepción inmediata de lo esencial de aquello que se observa. Constituye una forma de conocimiento directo caracterizada por la inmediatez y la contemporaneidad (Dowie y Elstein, 1999). Valgan como ejemplos de aplicación cotidiana del conocimiento intuitivo, las vivencias estéticas y de captación de valores. En suma, con mayor razón para el clínico, la intuición es una forma de pensamiento complementaria al razonamiento formalizado.

En el orden emocional e interpersonal, la empatía es la cualidad que permite inferir y comprender en profundidad aquello que el otro siente. La empatía es una propiedad, intuitiva y a la vez interpretativa, que permite la captación íntima de las emociones y sentimientos ajenos, así como su significación e importancia subjetiva (Sims, 2008). Dicha significación la capta el clínico por un proceso de transposición, imaginario e interpretativo, que adopta el marco interior de referencia del paciente, sin que por ello el clínico pierda la referencia de su propia identidad.

De algún modo la intuición, ese saber implícito -“saber tácito" lo llamó Polanyi (1967)- que junto al sentido común, forman parte del conocimiento ordinario el cual, sin ser ajeno a la razón, posee un sustrato empírico, de conocimiento previo y de experiencia importantes. El científico no lo desdeñará sino que sabrá incorporarlo al proceso de avanzar en el conocimiento. La intuición inspira un cierto paralelismo con la interpretación en el sentido de que ambas dan rápidamente con algo que no siendo inicialmente patente se vuelve manifiesto. Con ella se llega a una conclusión concreta sin que, por lo general, se tenga conciencia de todos los pasos lógicos intermedios (Damasio, 2006; Dowie y Elstein, 1999).

¿Pero cómo evaluar entonces la calidad de las intuiciones que aplica el evaluador clínico? La calidad de ellas dependerá de lo bien que se haya razonado con anterioridad; de lo bien que se hayan clasificado los acontecimientos de la experiencia pasada en relación con las emociones que los preceden y suceden; y también de lo acertadamente que se haya reflexionado sobre éxitos y fracasos de intuiciones manejadas con anterioridad (Damasio, 2006). El "insight", facilitado por la intuición, permite el discernimiento de lo propio, mientras que el "grasping" (captar) se refiere a la detección o aprehensión rápida del núcleo de sentido de una actuación ajena (Castilla del Pino, 1992). Esta capacidad se potencia con la empatía, que en última instancia no deja de ser, a su vez, la capacidad de inferir el sentimiento ajeno.

El término "insight" puede ser traducible no sólo como discernimiento rápido y comprensión penetrante, sino también como golpe de intuición, pues "ésta es una condición para [ese] golpe de vista [el "insight"] que, por definición, se considera acertado" (Castilla del Pino, 2004, p. 335). Aunque intuición, "insight" y "grasping" sean conceptos todavía insuficientemente estudiados, no por eso dejan de ser actuantes. De hecho, la maestría del profesional evaluador no es ajena a ellos y se refleja, por ejemplo, en su capacidad de manejar selectivamente mucha información, establecer inferencias bien controladas y mantener varios modos de considerar las cosas al mismo tiempo; todo ello sin interrumpir el flujo de la investigación conducente a confirmar o refutar las hipótesis de trabajo (Basoredo, 2011; Schön, 1998).

En suma, donde no es posible el acceso al conocimiento objetivo se requiere el concurso de una 
"subjetividad disciplinada" (Ericson, 1958). Esta actitud autocontrolada y precavida de objetivación, ha de conducir a someter las interpretaciones a prueba. Lo cual pasa por el refrendo del paciente y de otros informantes, así como el empleo de otros procedimientos alternativos, entre ellos el de la triangulación (Ibáñez, 1997). Otra de las maneras de contrastar las conclusiones interpretativas la ofrece el trabajo en grupo y con mayor garantía el trabajo en equipo multidisciplinar (Malcolm, 2004).

La realidad nos dice que el modo humano de procesar la información no es tan lógico y racional en tanto que la razón no puede dar cuenta del pensamiento, sino que en su lugar se utilizan heurísticos (Tversky y Kahneman, 1984). Estos son reglas y estrategias cognitivas, por lo general sencillas y fáciles, que sirven para decidir con rapidez y solucionar problemas, aunque con sesgos (González Labra, 2011). La cognición humana, más que lógica, es "psico-lógica" (Moreno y Peñacoba, 1996).

Los heurísticos sirven también y sobre todo para descubrir, también para comprender, y se insertan dentro del pensamiento inductivo y analógico, en contraposición a los desarrollos deductivos y analíticos más rigurosos. De hecho, el valor heurístico de una teoría reside en la fuerza que tenga para sugerir hipótesis verificables. Las interpretaciones actúan a modo de hipótesis imprescindibles en la buena marcha de las evaluaciones clínicas (Ibáñez, 1997; 2010).

Más adelante se aludirá a los errores interpretativos que la intuición y los heurísticos cognitivos conllevan, pero no se entenderá bien si no se parte de lo siguiente. No existen buenas interpretaciones que vengan establecidas de antemano, sino maneras idóneas de llegar a ellas guiados por una combinación de confiada osadía intelectual y prudencia ante el hecho mismo de interpretar (Gigerenzer, 2008).

\section{Niveles interpretativos}

Los datos piden ser interpretados, para lo cual el evaluador, en el marco de algún modelo teórico que le proporcione los elementos necesarios para hallar el significado psicológico de los datos, irá de los indicadores a los constructos.

Las inferencias interpretativas se efectúan a dos bandas:

a) Por parte del sujeto evaluado. La inferencia es un grado de transformación de la información tendente a otorgarle sentido que puede concentrarse no sólo en el lado del evaluador sino también en el de la parte evaluada. El sujeto evaluado en el proceso de suministrar información al evaluador efectúa algunas inferencias.

En este sentido se distinguen tres niveles de inferencia (Escalante, 2010; Ibáñez, 1997):

- De verbalización. Se refiere a contenidos informativos inmediatos que el sujeto evaluado comunica, espontáneamente o a requerimiento del evaluador, sin que medie de su parte elaboración interpretativa.

- De descripción. Se refiere a una información más elaborada que las meras verbalizaciones, donde se produce alguna descripción, cualitativa o cuantitativa, de dichas informaciones, lo que entraña en alguna medida de su parte valoración interpretativa.

- De explicación. Alude a un tipo de información muy elaborada, de intención explicativa, que como tal conlleva una importante carga interpretativa y el consiguiente riesgo de caer en error.

b) Por parte del evaluador. Se han diferenciado cuatro niveles de progresiva intensidad inferencial (Sundberg, Tyler y Taplin, 1973):

- Nivel de inferencia I. Concierne a datos que son directamente observables donde la conducta a evaluar es considerada como muestra del comportamiento habitual del sujeto evaluado, lo que ilustra acerca de lo que se espera que haga en condiciones parecidas. Este nivel bajo de inferencia es típico del modelo conductual.

- Nivel de inferencia II. Se efectúa una generalización empírica, de forma que la conducta a evaluar se relaciona con otra más amplia con la que habitualmente va junta y cuya relación está estudiada. Es propio del modelo conductual-cognitivo.

- Nivel de inferencia III. Aquí las afirmaciones teóricas van más allá de lo observable y necesitan un puente para llegar a lo empírico, las reglas de correspondencia. Las reglas de correspondencia permiten pasar del nivel teórico al empírico y viceversa. Este nivel de inferencia implica tomar la conducta a interpretar como signo de la existencia de determinado constructo -un rasgo de personalidad, por ejemplo- que (hipotéticamente) la explica. Así que en estos supuestos se parte de interpretar ciertos constructos que subyacen a los comportamientos evaluados como sucede con las inferencias habituales en los modelos teóricos de atributo y cognitivo.

- Nivel de inferencia IV. El concepto inferido de la conducta del sujeto se integra directamente, sin reglas de correspondencia, en una teoría de la 
personalidad en la que halla su sentido interpretativo profundo. Este tipo de salto inferencial excesivo es frecuente en el modelo psicoanalítico donde la interpretación como tal cobra un protagonismo muy alto. Hasta el punto de poder caer en el error al pasar del contexto de descubrimiento a las conclusiones sin detenerse en el contexto de justificación.

Tal como se ha visto, las inferencias no son fáciles de realizar con el debido rigor. Sin embargo, a la postre son necesarias, por más que se imponga la cautela y contención. Si todas las ciencias interpretan, la psicología no es ninguna excepción (Staats, 1997).

\section{Errores y sesgos interpretativos}

Las interpretaciones pueden errar por especulativas o por el efecto indeseado de diferentes sesgos del evaluador, quien interpreta apoyado en sus esquemas de conocimiento y disposiciones lo que percibe, lo que analiza y valora y, en general, el conjunto de elaboraciones que efectúa de las interpretaciones que maneja. El sesgo es un error que se comete de modo sistemático $\mathrm{y}$, en general, no consciente por lo que cuesta más corregirlo (Macrae y Bodenhausen, 2000). La activación de prejuicios y estereotipos, por ejemplo, asociados al envejecimiento (Bustillos, Fernández-Ballesteros y Huici, 2012), no sólo se ha comprobado que afectan al comportamiento de los pacientes, sino al de los mismos evaluadores, produciendo en ellos distorsiones interpretativas (Devine, 1989; Wheeler y Petty, 2001).

Como se ha dicho, los errores pueden colarse en distintos momentos de la cognición: a) procesos de percepción; b) procesos de tratamiento cognitivo de la información; c) conceptos afectados por la idea acerca de sí mismo; d) atribuciones causales; e) expectativas; f) contenidos de la memoria reprimidos, deformados, etc. Así, algunos ejemplos de errores que se cometen en las interpretaciones son los asociados a la simplificación de las situaciones, el olvido de datos fundamentales, la confusión respecto a lo que informan (por ejemplo, confundir correlación estadística con causalidad) y el hecho de considerar sólo aquellos datos más representativos o que se muestran más disponibles, etc. (Tversky y Kahneman, 1984).

Los errores cometidos pueden ser de tres tipos: a) Por interpretación excesiva. Se medirá por el valor absoluto (positivo o negativo) de la diferencia de valores asignados a cada dato por el evaluador clínico y los jueces expertos. b) Por omisión de interpretación. En este caso el evaluador clínico no asigna peso a ítems de información que sí son valorados como interpretables por los jueces expertos. c) Por signo inverso de los errores de interpretación. Siempre en relación al asignado por jueces expertos, si se desea aplicar la evaluación externa del trabajo del evaluador clínico (Maloney, Risko, Preston, Ansari y Fugelsang, 2010).

La percepción - por supuesto también la del evaluador-es un proceso activo, cognitivo, guiado por filtros (por ejemplo, aquello que interesa percibir, los esquemas cognitivos, la teoría implícita de la personalidad barajada, etc.) y mediatizado por inferencias. Además de los sesgos de los autoinformes, los del evaluador se producen en los tres niveles de percepción, organización de la información percibida e interpretación consiguiente. "Los prejuicios de percepción o la estrechez de concepción limitarán gravemente al intérprete en la variedad y extensión de sus interpretaciones" (Levy, 1971, p. 66).

Cada proceso cognitivo está cuajado en potencia de lagunas, errores y distintos sesgos. Entre estos últimos se pueden diferenciar: los determinados por aspectos sociales y culturales; los que acompañan a los procesos de inferencia como tales; y los asociados a características del evaluador (personalidad, conocimientos, estado de ánimo, expectativas, etc.). (Vázquez, 1995). Ni siquiera los datos de observación directa están libres de sesgos, dado que el perceptor no es neutral u objetivo, sobre todo si se apoya en teorías que tienden a ser especulativas. Un caso muy extremo a considerar es el que Castilla del Pino (1991) denomina "error diacrítico", que ocurre cuando se homologa una interpretación a una observación o a un dato evidente, tal como se manifiesta en la psicopatología del delirio.

Los errores de interpretación más frecuentes consisten en: forzar el proceso dando por supuestas en las relaciones una vinculación causa-efecto inexistente;

Efectuar saltos inferenciales subjetivos no justificados;

Ignorar datos determinantes que permitirían plantear en sus justos términos la interpretación;

Dejarse arrastrar por la expectativa que fuerza la impresión de coincidencia entre las hipótesis y los resultados reales, objetivos;

Tender a sobrestimar, en la explicación del comportamiento ajeno, los factores de personalidad y a subestimar los factores situacionales.

A la tendencia interpretativa de confirmar, sin la necesaria sustentación, las expectativas, preconcepciones, incluidas las hipótesis del evaluador, se la conoce como "sesgo confirmatorio" (Wason, 1960). Es de gran importancia su control en el procesamiento de la 
información, en particular durante la fase de justificación del proceso evaluador (Fugelsang, Stein, Green y Dunbar, 2004).

Otros sesgos a evitar en la interpretación son: las inconsistencias e incoherencias; las ambigüedades, que ocultan insuficiente claridad y precisión; los automatismos interpretativos, prematuros y superficiales, impuestos por el "sentido común", que conducen a destacar la aparente, pero inconsistente, "coherencia" de algunas interpretaciones; las infravaloraciones o supervaloraciones; los datos e ideas que se dan falsamente por hechos;) las deducciones y presuposiciones infundadas; las proyecciones subjetivizadoras; las generalizaciones abusivas; las atribuciones indebidas; la influencia del heurístico de representatividad, según el cual se tiende a evaluar la probabilidad de que A se asocie con B basándose en la similitud percibida entre A y B; y finalmente la influencia del heurístico de disponibilidad o tendencia a basar los juicios en lo que es más familiar o se conoce mejor; etcétera (Brodish y Devine, 2005; Dunlosky, Rawson y Middlenton, 2005).

En resumen, tan inevitable como que el evaluador tenga que interpretar las informaciones y datos que le llegan, es que éstos se vean más o menos afectados de insuficiencias, errores y sesgos de muy diferentes tipos. Algunos de ellos ya se han apuntado y cabe destacar la confianza excesiva en los propios juicios y creencias o sobrevaloración del pensamiento propio, que por sí mismo tiende a dificultar la objetividad y corrección de las interpretaciones.

Un procedimiento útil para llevar a efecto, en situaciones de formación y supervisión, la tarea de evaluar el grado de competencia que el evaluador clínico recién iniciado posee al interpretar, es el panel de expertos. Estos jueces tendrán acceso a las cogniciones y metacogniciones del sujeto a evaluar a través de la información cualitativa que les llegue por medio de la técnica cognitiva del "pensamiento-en-voz-alta". A través de ella conocerán la forma en que el clínico en cuestión percibe, analiza, interpreta y enjuicia los distintos reactivos, con la posibilidad de valorar sus aciertos y errores. Podría hacerse en vivo (con algunas reservas) o en diferido (con limitaciones producidas por el rendimiento de la memoria...) (Cohen y Swerdlik, 2001).

\section{La integración interpretativa}

El proceso de integración de datos, paralelo al de interpretación, consiste en pasar de la multiplicidad de datos - debidamente filtrados, analizados, organizados, relacionados, asociados - a su progresiva reducción, vía agrupación y síntesis de los más significativos hallados. En la primera fase analítica la reducción exige organizarlos en categorías manejables, aclarar su utilidad, sintetizar y comparar. La segunda fase de reducción pasa por seleccionar, focalizar, abstraer y transformar los datos e informaciones disponibles. Este proceso de integración interpretativa facilita la comprensión de la estructura y significado global. La cantidad y la complejidad de los datos que habitualmente tiene a disposición el evaluador, a menudo exceden su capacidad integradora (Haynes, 1994). Según recuerda este autor, es más difícil extraer inferencias de las variables de nivel superior, que son las que se barajan en las fases avanzadas del proceso evaluador. En todo caso, existen distintos niveles de integraciones.

La interpretación como tal va unida a la integración de datos, de modo que en el proceso de evaluación, el desdoblamiento atomizador provocado por el análisis inicial derive en síntesis global y recuperación de la realidad unitaria del sujeto evaluado. Los datos a integrar incluirán aquellos que, aunque parciales, fragmentarios y en apariencia periféricos, resulten útiles para respaldar la evaluación de cara a la emisión del juicio diagnóstico, etc. Por lo general, los errores al respecto se deben menos al defecto de los datos que a los fallos en la manipulación de los mismos y a errores que se cometen al integrarlos e interpretarlos (Elstein y Bordage, 1984). Finalmente, la información disponible para el evaluador clínico ha de ser -además de suficiente y relevante- válida, significativa y consistente.

La forma emergente de trabajo multidisciplinar si bien permite abordar en su complejidad bio-psicosociocultural (Montealegre, 2005) la actividad humana y más en particular los problemas estudiados en el área clínica, exige mayor capacidad de integración de la multiplicidad de datos provenientes de campos de conocimiento distintos. Las perspectivas de los profesionales que confluyan en la evaluación del caso clínico se esforzarán para llegar a síntesis integradoras idiográficas de los factores relevantes, y que por supuesto integren asimismo las perspectivas de los clínicos y la de los pacientes (Mezzich et al., 2002).

\section{Recomendaciones finales para interpretar en clínica}

La interpretación ha de apoyarse en el conocimiento científico y en una forma científica de trabajar. De modo 
que una interpretación filosófica o especulativa, que se limite a dar cauce a una intuición alejada de la evidencia empírica, no puede ser conclusiva si no está justificada al modo de las hipótesis de trabajo. Por lo demás, es indudable que con frecuencia pueden servir, además, como estímulo del pensamiento. Por consiguiente, además del esfuerzo de evitar caer en errores y sesgos, conviene tener presentes algunas pautas, directrices o recomendaciones útiles que garanticen la calidad científica (Fernández-Ballesteros y Calero, 2004; Newman, 2010; Rodolfa et al., 2005). Las recomendaciones que siguen atañen específicamente al proceso cognitivo de interpretar correctamente.

La responsabilidad inherente a la interpretación es una tarea que el clínico no puede delegar en nada (método, técnica...) ni en nadie. Aprender a pensar de forma empírica le alertará sobre la necesidad de contar con suficiente evidencia para la interpretación. En caso de duda sobre si efectuar o no una interpretación, se aconseja no hacerla o ajustar su nivel hacia abajo. Obviamente, toda forma de conocimiento ha de sustentarse en la lógica del procedimiento y en la convicción íntima del agente.

Valorar el alcance, posibilidades, validez, limitaciones... de los datos de base que vayan a ser interpretados. En líneas generales, conforme el comportamiento a evaluar sea más simple, observable en forma directa, dotado de significado patente y en apariencia menos relevante en relación a la explicación o consecuencias del problema en estudio, en principio, se requerirá menor carga e intensidad interpretativas.

Tener siempre en cuenta en toda su complejidad las interacciones entre datos (cuantitativos y cualitativos) y de éstos con los antecedentes y el contexto donde se producen.

Extraer el máximo de posibilidades que proporcione la teoría o modelo teórico de referencia, pero sin forzarlos en ningún caso. La profundidad de la interpretación debe ser apropiada a la calidad (objetividad, validez...), relevancia de los datos disponibles y objetivos.

Para poder emitir un diagnóstico y evaluación correctos es más útil disponer de una buena integración, valoración e interpretación de la información que empeñarse en acumular demasiada información. El buen profesional sabe qué información necesita y cuándo es relevante.

Evitar en lo posible el manejo de información ambigua en tanto que exacerba la urgencia interpretativa, el riesgo de imprecisión al efectuarla y conduce a resultados subjetivos carentes de fiabilidad. Apoyarse en lo posible en datos objetivos, limitando al máximo las inferencias de alto nivel; así como posponer en el tiempo, el momento interpretativo a fin de evitar o reducir el efecto multiplicador de los sesgos producidos en fases interpretativas tempranas.

Buscar la coherencia en las concatenaciones e integraciones que se hagan de las interpretaciones puntuales de los datos, antes de integrarlas en la fase final que precede a la emisión de juicios diagnósticos, pronósticos y otros.

Deben darse suficientes evidencias para apostar por una determinada interpretación; evidencias que sean, además, recurrentes y concurrentes, convergentes. Aprender a distinguir entre impresiones subjetivas y evidencia objetiva. Ha de tenerse siempre presente la condición probabilística de las interpretaciones.

Las interpretaciones deben pasar un proceso interno de validación que lleve a considerarlas primero como hipotéticas y ser probadas antes de su aceptación. Contrastar dichas hipótesis conlleva, en sentido estricto, considerar las hipótesis rivales que pudieran refutarlas; esto es un trámite que tiende a evitarse.

Por último, a modo de recomendación general, subrayar que el hecho de remitirse a la evaluación clínica basada en la evidencia impulsa las metacogniciones del profesional y le aporta, entre otras, la ventaja de minimizar la subjetividad y sesgos de las interpretaciones, lo que permite incrementar la validez y rigor de las mismas y por ende de las decisiones que se tomen de cara a la intervención.

La ilusión del profesional que empieza y su deseo de demostrar que se halla capacitado para enfrentar con eficiencia la compleja tarea que lleva entre manos, en un caso; y la precipitación o urgencia del profesional avezado, excesivamente "seguro" de sus intuiciones, en otro caso; pueden conducir a que, en ocasiones, tanto unos como otros no se tomen el tiempo y cuidados necesarios que exige el trabajo de calidad.

\section{Conclusión}

En este artículo se ha recordado que la psicología, como cualquier otra ciencia, es interpretativa y que por ello moviliza este poderoso recurso cognitivo capaz de potenciar el conocimiento. Toda interpretación incluye un componente, mayor o menor, de subjetividad por lo que es prácticamente imposible demostrar la corrección o validez definitiva de la misma; si bien varía su calidad, unas interpretaciones son más contenidas, lúcidas y rigurosas, más certeras y científicas, que otras. Se ha mostrado que interpretar conlleva procesos cognitivos de gran complejidad y riesgo. 
El acierto para interpretar compone una competencia general que respalda la eficacia del profesional clínico, no sólo en sus tareas en la evaluación, sino también en las de intervención. Sin embargo, no siempre se le presta la debida atención en los programas de formación y cualificación. A nuestro entender el pulso principal se plantea entre la necesidad de objetivar, de atenerse a la evidencia; y la de profundizar y asegurar el acceso a conocimientos personalizados y de significación completa.

El profesional experto se plantea las cuestiones de modo oportuno, correcto, posee más respuestas, pero también es capaz de hacerse más preguntas, todo lo cual le capacita para interpretar. En este sentido habrán de evitarse los errores y sesgos interpretativos. Para facilitar esto se han aportado algunas pautas que ayudan a mejorar la calidad de las interpretaciones del evaluador clínico.

\section{Referencias}

Adams-Webber, J. R. (2003). Cognitive complexity and confidence in evaluating self. Journal of Constructivist Psychology. 16(3), 273-279.

Adams, F., y Aizawa, K. (2010). The bounds of cognition. Oxford, UK.: Wiley-Blackwell.

Allport, G. (1977). La personalidad. Su configuración y desarrollo. Barcelona: Herder.

Arias, A. (2012). Avatares del paradigma conexionista. Ciencia Cognitiva, 6(1), 13-16.

Banyard, P., Cassells, A., Green, P., Hartland, J., Hayes, N., y Reddy, P. (1995). Introducción a los procesos cognitivos. Barcelona: Ariel.

Basoredo, C. (2011). Una perspectiva y un modo de explicar la competencia desde el ámbito del desempeño de tareas. Anales de Psicología, 27(2), 457-472.

Bennett-Levy, J. (2006). Terapist skills: A cognitive model of their acquisition and refinement. Behavioural and Cognitive Psychotherapy, 34, 57-78.

Brodish, A. B., y Devine, P. G. (2005). The dynamics of prejudice, stereotyping, and intergroup relations: Intrapersonal and interpersonal processes. Social Psychological Review, 7, 54-70.

Bruner, J. (1990) Actos de significado. Más allá de la revolución cognitiva. Madrid: Alianza.

Bustillos, A., Fernández-Ballesteros, R., y Huici, C. (2012). Efectos de la activación de etiquetas referidas a la vejez. Psicothema, 24(3), 352-357.

Butler, A. C., Champan, J. E., Forman, E. M., y Beck, A. T. (2006). The empirical status of cognitive-behavioral therapy: a review of meta-analysis. Clinical Psychology Review, 26, 17-31.

Caro, I. (2011). Hacia una práctica eficaz de las psicoterapias cognitivas. Bilbao: Desclée de Brouwer.

Castilla del Pino, C. (1991). Crítica a la razón psicopatológica. En C. Castilla del Pino y J. M. Ruiz-Vargas (Eds.), Aspectos cognitivos de la esquizofrenia (pp. 11-33). Madrid: Trotta.

Castilla del Pino, C. (1992). Interpretación, interpretado, intérprete. Theoría, 16-17-18, tomo B, 1353-1366.
Cohen, R. J. y Swerdlik, M. E. (2001). Pruebas y evaluación psicológicas. Introducción a las pruebas y a la medición (4a ed.). México: McGraw-Hill/Interamericana.

Cormier, W. H. y Cormier, L. S. (1994). Estrategias de entrevista para terapeutas $\left(2^{\mathrm{a}}\right.$ ed.). Bilbao: Desclée de Brouwer.

Damasio, A. (2006). El error de Descartes. Barcelona: Crítica.

Devine, P. G. (1989). Stereotypes and prejudice: Their automatic and controlled components. Journal of Personality and Social Psychology, 56(1), 5-18.

Domingo, J. M. (2005). Pensar en contexto. En F. Gabucio (Coord.), Psicología del pensamiento (pp. 269-293). Barcelona: UOC.

Dowie, J. y Elstein, A. (Eds.) (1999). Professional judgment. A reader in clinical decision making. Cambridge, UK: Cambridge University Press.

Dunkley, D. M., Blankstein, K. R., y Segal, Z. V. (2010). Cognitive assessment: Issues and methods. En K. S. Dobson (Comp.), Handbook of cognitive-behavioral therapies (pp. 133-171). Nueva York: Guilford Press.

Dunlosky, J., Rawson, K. A., y Middlenton, E. L. (2005). What constrains the accuracy of metacomprehension judgments? Testing the transfer appropriate monitoring and accessibility hypotheses. Journal of Memory and Language, 52(4), 551-556.

Eco, U. (2009). Los límites de la interpretación. En: U. Eco, Cultura y Semiótica (pp. 55-83). Madrid: Círculo de Bellas Artes.

Eco, U. (2012). Hay más cosas que no se pueden decir. En torno al Realismo Negativo. Revista de Occidente, 373, 5-25.

Efklides, A. (2008). Metacognition, defining its facets and levels of functioning in relation to self-regulation and co-regulation. European Psychologist, 13(4), 277-287.

Elman, N. S., Illfelder-Kaye, J., y Robiner, W. N. (2005). Professional development: training for professionalism as a foundation for competent practice in psychology. Professional Psychology. Research and Practice, 36, 367-375.

Ericson (1958). Citado en Schön, D. El Profesional Reflexivo, (p. 112). Barcelona: Paidós.

Escalante, E. (2010). Métodos de análisis de las verbalizaciones: ontologías y proceso de abducción. Subjetividad y Procesos Cognitivos, 14(2), 50-69.

Evans, I. (1993). Constructional perspectives in clinical assessment. Nueva York: Wiley.

Fernández-Ballesteros, R., De Bruyn, E. E. J., Godoy, A., Hornke, L. F., Ter Laak, J., Vizcarro, C., Westhoff, K., Westmeyer, H., y Zaccagnini, J. L. (2001). Guidelines for the Assessment Process (GAP): A proposal for discussion. European Journal of Psychological Assessment, 17(3), 187-200. (Trad. de Godoy, A. (2003). Papeles del Psicólogo, 84, 58-70).

Fernández-Ballesteros, R. y Calero, M. D. (2004). Garantías científicas y éticas de la evaluación psicológica. En R. FernándezBallesteros (Ed.), Evaluación psicológica. Conceptos, métodos y estudio de casos (pp. 121-158). Madrid: Pirámide.

Fernández-Ballesteros, R., Márquez, M. O., Vizcarro, C. y Zamarrón, M. D. (2011). Buenas prácticas y competencias en evaluación psicológica. Madrid: Pirámide.

Fugelsang, J., Stein, C., Green, A., y Dunbar, K. (2004). Theory and data interactions of the scientific mind: Evidence from the molecular and the cognitive laboratory. Canadian Journal of Experimental Psychology, 58, 132-141.

Fumero, A., Santamaría, C y Johnson-Laird, P. (2010). Formas de pensar: efecto de la personalidad en el razonamiento. Psicothema, 22(1), 57-62.

Gabucio, F. (2004). De los problemas del método científico. Revista de Psicología y Humanidades EPSYS, 2-8. 
Gigerenzer, G. (2008). Decisiones instintivas. La inteligencia del inconsciente. Barcelona: Ariel.

Godoy, A. (1996). Toma de decisiones y juicio clínico. Madrid: Pirámide.

Godoy, A. y Gavino, A. (1999). Conocimiento científico-básico y conocimiento clínico-aplicado: su papel en la realización de diagnósticos clínicos. En F. Silva (Coord.), Avances en evaluación psicológica (pp. 77-125). Valencia: Promolibro.

González Labra, M. J. (2011). Introducción a la Psicología del Pensamiento. Madrid: Trotta.

Haynes, S. N. (1994). Juicio clínico y diseño de programas de intervención conductual: Estimación de la magnitud de los efectos de la intervención. Psicología Conductual, 2(2), 165-184.

Hofer, B. K. y Pintrich, P. R. (2002). Personal epistemology. The psychology of beliefs about knowledge and knowing. Mahwah, NJ: Erlbaum.

Ibáñez, C. (1997). Evaluación psicológica. Lecciones introductorias. Bilbao: Servicio Editorial UPV.

Ibáñez, C. (2003). De la teoría psicológica a los datos empíricos: camino de ida y vuelta. Psiquis, 4(6), 30-40.

Ibáñez, C. (2005). Más allá de la clasificación diagnóstica: la calidad de la asistencia clínica. Psiquis, 26(2), 37-49.

Ibáñez, C. (Ed.) (2010). Técnicas de autoinforme en evaluación psicológica. La entrevista clínica. Bilbao: Servicio Editorial UPV.

Ibáñez, C. y Maganto, C. (2009). El proceso de evaluación clínica: cogniciones del evaluador. Summa Psicológica UST, 6(1), 81-99.

James, I. A., Southem, L., y Blackburn, I. M. (2004). Schemas revisited. Clinical Psychology and Psychoterapy, 11, 369-377.

Kaslow, N. J. (2004). Competencies in professional psychology. American Psychologist, November, 774-781.

Lee, J., y Truex, D. P. (2000). Cognitive complexity and methodical training: enhancing or suppressing creativity. Proceeding of the 33rd Hawai International Conference on System Sciences.

León, J. A. y Pérez, O. (2003). Taxonomías y tipos de inferencias. En J. A. León (Ed.), Conocimiento y discurso. Claves para inferir y comprender (pp. 45-66). Madrid: Pirámide.

Levy, L. H. (1971). La interpretación. México: F. C. E.

Macrae, C. N., y Bodenhausen, G. V. (2000). Social cognition: Thinking categorically about others. Annual Review of Psychology, 51, 93-120.

Malcolm, G. (Ed.) (2004). Multidisciplinary approaches to visual representations and interpretations. (Studies in multidisciplinarity, vol. 2). Amsterdam, The Netherlands: Elsevier.

Maloney, E.A., Risko, E.F., Preston, F., Ansari, D., y Fugelsang, J. (2010). Challenging the reliability and validity of cognitive measures: The case of the numerical distance effect. Acta Pyschologica, 134, 154-161.

Marina, J. A. (1996). Teoría de la inteligencia creadora. Barcelona: Anagrama.

McClelland, J. L. (2010). Emergence in cognitive science. Topics in Cognitive Science, 2, 751-770.

Mezzich, J. E., Berganza, C. E., Cranach, M., Jorge, M. R., Kastrup, M. C., Murthy, R. S., Okasha, A., Pull, Ch., Sartorius, N., Skodol, A., y Zaudig, M. (2002). Directrices Internacionales para la Evaluación Diagnóstica (IGDA). Nueva York: Asociación Mundial de Psiquiatría (AMP).

Montealegre, R. (2005). La actividad humana en la psicología histórico-cultural. Avances en Psicología Latinoamericana, 23, 33-42.

Moreno y Peñacoba (1996). Citado en A. Fierro (Ed.) (1996), Manual de psicología de la personalidad. Barcelona: Paidós.

Morin, E. (2001). Los siete saberes necesarios para la educación del futuro. Barcelona: Paidós.
Newman, C. F. (2010). Competency in conducting cognitive-behavioral therapy: Foundational, functional, and supervisory aspects. Psychotherapy, 47, 12-19.

Nisbet, J. y Shucksmith, J. (1986). Learning strategies. London: Routiedge \& Kegan Paul. (Trad. de Bermejo, A. (1987), Estrategias de aprendizaje. Madrid: Santillana/Aula XXI).

O’Brien, W. H. y Haynes, S. N. (1997). Análisis funcional de la conducta. En G. Buela -Casal y J. C. Sierra (Eds), Manual de evaluación psicológica. Fundamentos, técnicas y aplicaciones (pp.493-521). Madrid: Siglo XXI.

Patel, V. L., y Groen, G. J. (1992). Cognitive framework for clinical reasoning: Application for training and practice. En D. A. Evans y V. L. Patel (Eds.), Advances models of cognition for medical training and practice (pp. 193-211). Berlin: Sringer-Velag.

Pintrich, P. R. (2002). The role of metacognitive knowledge in learning, teaching, and assessing. Theory Into Practice, 41(4), 219-225.

Polanyi, M. (1967). The tacit dimensión. London: Routledge \& Paul.

Prins, F. J., Nadolski, R. J., Berlanga, A. J., Drachsler, H., Hummel, H. G. K., y Koper R. (2008). Competence Description for Personal Recommendations: The importance of identifying the complexity of learning and performance situations. Educational Technology \& Society, 11(3), 141-152.

Rodolfa, E., Bent, R., Eisman, E., Nelson, P., Rehm, L., y Ritchie, P. (2005). A cube model for competency development: Implications for psychology educators and regulators. Professional Psychology: Research and Practice, 36, 347-354.

Rodríguez Rebustillo, M. y Bermúdez, R. (2004). Hacia una metodología para condicionar la construcción del conocimiento científico. Revista Cubana de Psicología, 21(3), 206-213.

Roe, R. (2002). What makes a competent psychologist? European Psychologist, 7(3), 192-202.

Romo, M. (2008). Epistemología y psicología. Madrid: Pirámide.

Sandi-Urena, G. S. (2009). Desing and validation of a multimethod assessment of metacognition and study of the effectiveness of metacognitive interventions. Dissertation Abstracts International: Section B: The Sciences and Engineering, 69(8-B), 4717.

Schön, D. A. (1998). El profesional reflexivo. Barcelona: Paidós.

Shinamura, A. P. (2000). Toward a cognitive neuroscience of metacognition. Consciousness and Cognition, 9, 313-323.

Sims, A. (2008). Sintomas mentales. Madrid: Triascastela.

Staats, A. W. (1997). Conducta y personalidad: conductismo psicológico. Bilbao: Desclée de Brouwer.

Sundberg, N. D., Tyler, L., y Taplin, J. R. (1973). Clinical Psychology: Expanding Horizons. Nueva York: Prenctice Hall.

Tversky, A. y Kahneman, D. (1984). Juicio en situación de incertidumbre: Heurísticos y riesgos. En M. Carretero y J. A. García Madruga (Eds.), Lecturas de psicología del pensamiento (pp. 169-181). Madrid: Alianza.

Vázquez, C. (1995). Limitaciones, errores y sesgos en el procesamiento de la información: la ficción de la teoría del "hombre científico". En M. D. Avia y M. L. Sánchez-Bernardos (Eds.), Personalidad: Aspectos cognitivos y sociales (pp.185-225). Madrid: Pirámide.

Wason, P.C. (1960). On the failure to eliminate hypotheses in a conceptual task. Quarterly Journal of Experimental Psychology, 12, 129-140.

Wheeler, S. C., y Petty, R. E. (2001). The effects of stereotype activation on behavior: A review of possible mechanisms. Psychological Bulletin, 127(6), 797-826.

Yela, M. (1991). Unidad y diversidad de la Psicología. En J. Mayor y J. L. Pinillos (Eds.), Tratado de Psicología General. Historia, teoría y método (pp. 71-92). Madrid: Alhambra. 